\title{
How many fish could be vocal? An estimation from a coral reef (Moorea Island)
}

\author{
Eric Parmentier ${ }^{1, *}$, Frédéric Bertucci ${ }^{2,3}$, Marta Bolgan ${ }^{1}$ \& David Lecchini ${ }^{3,4}$ \\ ${ }^{1}$ Université de Liège, Laboratoire de Morphologie fonctionnelle et évolutive, FOCUS, \\ Institut de Chimie - B6c, Sart Tilman, Liège, 4000, Belgium. \\ ${ }^{2}$ Biology of Aquatic Organisms and Ecosystems (Unit BOREA), Université des Antilles- \\ MNHN-SU-UCN-CNRS-IRD, Pointe-à-Pitre, Guadeloupe. \\ ${ }^{3}$ Laboratoire d'Excellence « CORAIL », BP 1013, 98729, \\ Papetoai, Moorea, French Polynesia. \\ ${ }^{4}$ PSL Research University: EPHE-UPVD-CNRS, USR3278 CRIOBE, BP 1013, 98729, \\ Papetoai, Moorea, French Polynesia. \\ "Corresponding author: E.Parmentier@uliege.be
}

\begin{abstract}
A recurrent question arising in fish bioacoustics research concerns the number of vocal fish species that may exist. Although it is not possible to provide a precise globally valid number, an estimation based on recordings already collected at coral reefs (Moorea) and on morphological approaches indicates that approximately half of the fish families of this particular environment has at least one known soundproducing species. In light of this, acoustic behaviour should be fully considered in biology, ecology and management plans as it may provide information on a consistent portion of fish biodiversity. Fish bioacoustics has switched from anecdotal reports to long-term, large-scale monitoring studies, capable of providing high resolution information on fish populations' composition and dynamics. This information is vital for successful management plans in our quickly changing seas.
\end{abstract}

Keywords. Acoustic, biodiversity, monitoring, sonic, teleost.

PARMENTIER E., BertuCCI F., BOLGAN M. \& LeCChini D. (2021). How many fish could be vocal? An estimation from a coral reef (Moorea Island). Belgian Journal of Zoology 151: 1-29. https://doi.org/10.26496/bjz.2021.82

\section{Introduction}

In the last decades, increasing human pressure on marine ecosystems and resources, driven by demographic and economic growth, as well as by the diversification and intensification of maritime activities, poses unprecedented threats to global biodiversity and to the overall health of our seas. Fishing has always represented a central economic activity that has provided livelihood opportunities to hundreds of thousands of people while shaping the cultural fabric of coastal towns and communities. Today, the sea plays a central role, being increasingly pivotal for the sustainable development of many regions. The main challenge is 
to find new monitoring approaches capable of quickly providing high-resolution information on marine ecosystems and communities' status and health.

All teleost species may produce sounds if we include feeding, swimming, splashing and jumping noises (ROUNTREE et al. 2018). However, these sounds can be considered as incidental or as by-products of fish activities and are not species- or family-specific. In order to monitor fish species in the wild, based on the sounds they naturally emit, the sampling effort should focus only on sounds which are species-specific. This is the case for communicative sounds, i.e., sounds which are intentionally made by fish during social interactions to elicit a behavioural response in the receiver that is advantageous (at least) for the sender (BRADBURY \& VEHRENCAMP 1998). Fish sound production was first mentioned by Aristotle and it has been known and exploited by fishermen for centuries (LOBEL et al. 2010). Fishermen from different regions worldwide locate fish aggregations by listening to their sounds. For example, in the Gironde estuary, the long-standing knowledge about the meagre (Argyrosomus regius) vocal abilities has been used by fishermen to locate large aggregations of this species during its spawning period (LAGARDĖRE \& MARIANI 2006). However, the idea that most underwater organisms (except whales and dolphins) would be unable to produce sounds and live in a silent environment is still very popular, maybe aided by a misapprehension derived from the documentary "The Silent World" (COUSTEAU \& DUMAS 1953). Some decades ago, only few fish species were considered to rely on acoustic communication (MOULTON 1958, 1963; FISH \& MOWBRAY 1970; LOBEL et al. 2010). Today, fish acoustic communication should be considered as an important aspect of teleost social behaviour across a wider taxonomic spectrum; fish sounds have indeed been reported in several, unrelated taxa inhabiting different aquatic habitats. Fish sounds have been recorded in freshwater streams, rivers and lakes, as well as in temperate and tropical coastal areas and also in the deep-sea (FINE \& PARMENTIER 2015). Acoustic signals mediate fish social interactions in a wide range of activities which are crucial for their survival, such as competitive feeding, distress or alarm situations, conspecific identification, courtship and agonistic interactions, mate choice, mate quality assessment and coordination of gamete release (AMORIM et al. 2015). Collectively, the increasing number of studies describing the vocal abilities of marine fish points to acoustic communication as an important aspect of teleost biology, which mediates social interactions during pivotal activities for fish survival and fitness. Reliance on acoustic communication may be of even greater importance for nocturnal species or for species living in deep sea habitats (RUPPÉ et al. 2015; DESIDERÀ et al. 2019).

The Actinopterygii comprises 488 families (VAN DER LAAN \& FRICKE 2019) and around 34200 species (FROESE \& PAULY 2019). In some taxa, all or almost all species have the ability to produce sounds for social purposes (FINE \& PARMENTIER 2015). Conversely, large fish families may be mostly mute since none, one or only a few species have been reported to produce socially relevant sounds (FINE \& PARMENTIER 2015). However, cycles of sound-producing muscle hypertrophy during mating seasons (CONNAUGHTON et al. 1997) imply that some species are unlikely to be vocal throughout the rest of the year, making the adage "absence of evidence is not evidence of absence" particularly relevant.

The assessment of the total number of vocal fish species could be achieved by counting the number of species for which sounds have been effectively recorded. This number should be updated each time a new study on the subject is published. Moreover, this number could be further improved by using morphological data, which allow to confidently infer the ability to emit sounds even in species that have not yet been recorded, as long as they have been shown either to possess the required mechanisms or be closely related to a vocal species (FRÉDÉRICH et al. 2014; RAICK et al. 2018). The estimation of an exact globally valid number of vocal fish species would be extremely time consuming and likely ineffective if all fish taxa present worldwide are surveyed by using the above-mentioned approach. A more effective strategy would be to apply this approach to specific habitats first. Here, we focus on one of the most acoustically and taxonomically diverse, as well as most studied, aquatic environments, i.e., coral reefs. To provide a first response to the question "how many fish are vocal?", we calculated the ratio of calling teleost species to 
the total number of species in the coral reefs of Moorea Island (French Polynesia), where fish acoustic communication has been investigated for more than 20 years. We postulate that our approach to estimate the ratio of vocal species, which we calculated for a localised, well-known fish vocal community, can be applied to other marine habitats and eventually be upscaled to estimate a global ratio of vocalising fish species. Under the assumption that environmental pressures and taxonomic composition have acted similarly on acoustic diversity in different biotopes, we would expect that ratios being precisely calculated for a selected number of different biotopes with our approach could approximate the ratio of other different environments and provide a rough but coherent estimation of the total number of potential vocal fish species.

In coral reefs, fish acoustic behaviours are prominent as many species produce sounds during agonistic interactions with competitors, responses to predators or threats, and during courtship and spawning. Worldwide, 179 fish families occupy coral reefs and adjacent marine habitats. Sounds were first reported in species of 48 (27\%) of these families (LOBEL et al. 2010). However, these authors considered all sounds, including swimming and feeding sounds that could be by-products of movement and food processing. Boyle and Tricas conducted a pioneering study to estimate the ratio of vocal species in Hawaiian reefs (TRICAS \& BOYLE 2014). They estimated that 45 species out of 96 (from 12 families out of 24) produced sounds in several behavioural contexts. This means that half of the detected families were represented by at least one vocal species. However, Boyle and Tricas' methodology was based on scuba diving performed during daytime, which therefore excludes many species that are mostly vocally active during night time hours and/or that are behaviourally cryptic (RUPPÉ et al. 2015; PICCIULIN et al. 2019). This approach also overlooked small and cryptic species such as gobies or blennies, which have sonic representatives (DE JONG et al. 2007; MALAVASI et al. 2012). In fact, a total of 481 species (not 96) can be found in Hawaiian reefs (FROESE \& PAULY 2019) and hence it is likely that the number of vocal species was underestimated.

The recently published complete list of shore fishes from French Polynesia (SIU et al. 2017) was used here to estimate the ratio of calling species, genera and families (Table 1). In Moorea, 18 of the 66 fish families (27\%) possess species for which sound production for communication purposes has been demonstrated (Table 1). Obviously, many species living in this area have not yet been recorded, i.e., they have not been investigated yet in terms of vocal abilities. This implies that the number of calling species is most probably larger than estimated. Species from French Polynesia that belong to genera such as Platax $s p$. (Ephippididae), Hyporhamphus sp. (Hemiramphidae), Polydactylus sp. (Polynemidae) and Caranx sp. (Carangidae) possess vocal co-generics in other parts of the world (FISH \& MOWBRAY 1970; LOBEL et al. 2010) supporting the possibility that species from Moorea are also capable of sound production. If we include these four additional taxa, the ratio of families having at least one vocal species would be up to $33 \%$.

Moreover, it is interesting to consider nine additional fish families from French Polynesia (Aulostomidae, Bythidae, Blenniidae, Diodontidae, Gobiidae, Hemiramphidae, Ophidiidae, Scorpaenidae, Syngnathidae) which hold species that are potentially vocal for two reasons. First, some of them possess anatomical features supporting sound production (HALLACHER 1974; PARMENTIER et al. 2010; 2013; FINE \& PARMENTIER 2015) and second, acoustic communication has been proven in species (MOULTON 1958, 1963; LOBEL et al. 2010; TRICAS \& BOYLE 2014) being from the family but not from the same genus. Based on these assumptions, we can reasonably suggest that these nine families (Table 1) should also be added to the list of soniferous fish families from Moorea. In this case, the ratio of vocal families further increases to $47 \%$ (32 out of 66). Additional data are obviously needed to identify and validate calling species identity within these families and in others. At the current state of art, we know that there are more calling species than those currently identified, because most of the sounds recorded in the wild using passive acoustics have not yet been attributed to a species (JUBLIER et al. 2019; BERTUCCI et al. 2020). 
Considering the lower taxonomic genus level, $22 \%$ of the 241 fish genera inhabiting Moorea's reef include species known to use acoustic communication (Table 1). Although one fifth is already an important ratio, we suspect that this number is probably still an underestimation if we follow the same reasoning that was applied for the families. Fish species from ten genera which are present in Moorea have been recorded in other parts of the world (Table 1). Adding these genera to our assessment increases the ratio of fish genera having vocal species to $26 \%$.

We can try to tackle this argument also in another way. The 31 (or 47\%) fish families of Moorea that possess calling fish altogether contain 161 genera (Table 1). If all genera that belong to the 31 aforementioned families have sonic species, $67 \%$ of the genera found in Moorea could then have at least one representative vocal species. However, acoustic abilities have not yet been indicated in all genera within these families and it is not always easy to make predictions. For example, many if not all Carapidae, Holocentridae and Pomacentridae species probably make sounds because they all possess the required acoustic sonic mechanisms (FINE \& PARMENTIER 2015). In other families, such as Blenniidae or Gobiidae, the sonic ability has been shown in certain genera only (DE JONG et al. 2007; MALAVASI et al. 2012) and further studies still have to be conducted to investigate other genera. This comparison highlights the need of additional tests to better assess the number of fish genera having calling species.

Coral reefs are characterised by rich habitat complexity and biodiversity; here, fishes have evolved amazing colour patterns. Although environmental conditions of coral reefs should favour visual communication, the ratio of calling species to not calling species is quite significant. Importantly, fish acoustic diversity peaks during night-time hours, i.e., when visual communication is not possible (RUPPÉ et al. 2015; BERTUCCI et al. 2020). This suggests that acoustic communication should be important also under other conditions in which dependence on visual communication is impaired, such as murky and dark environments as well as the deep-sea. Although many deep-sea fish species are likely to emit sounds as they possess the required anatomical structures, empirical data for this biotope are still too scattered to provide robust estimations on the ratio of vocal to not vocal fish species (BOLGAN \& PARMENTIER 2020).

\section{Conclusion}

Providing a precise number of vocal fish species is currently impossible as it would require an alienating and time-consuming effort to collect, identify, and record all worldwide distributed species that have not yet been recorded. We here humbly attempted to estimate a first ratio of vocal species by taking advantage of a unique combination of extensive recordings and monitoring of the ichthyologic community of the coral reef of Moorea. Half of the families and around a third of the genera in Moorea have been estimated to contain species being able to produce communicative sounds. Even if approximated and limited to a single habitat, these ratios emphasise the importance that calling behaviour may have in the biology of many more fishes than previously thought. Biodiversity assessments remain as one of the most difficult challenges encountered by ecologists and conservation biologists. This growing need for biodiversity assessments raises questions of whether current monitoring techniques are appropriate and efficient. Indeed, biodiversity assessment tools should effectively detect the highest possible number of species, including cryptic ones, in order to provide high resolution information on fish diversity. By taking advantage of the sounds produced by fish species, passive acoustic monitoring has demonstrated its potential to evaluate the diversity of coral reef fish, based on the vocal activities of their numerous inhabitants. The present study clearly supports that fish bioacoustics should be considered as an important tool in marine conservation and monitoring studies. Acoustic behaviour should be fully included in the biology and ecology of teleosts and in future conservation studies of marine ecosystems. However, additional studies are still required to confirm the same ratio in other habitats. Although at present risky, extrapolation of our observations to all teleost species would indicate that up to half of the world's fish families (i.e., 244 out of 448) could possibly possess vocal species. 


\section{TABLE 1}

List of the fishes present in Moorea waters (French Polynesia) with information related to acoustic communication. VSM: identified vocal species from Moorea. VG: genus of coral reef family having at least one identified vocal species. PVG: genus of coral reef having potentially (based on literature) one vocal species. Family in yellow: at least one identified and recorded calling species. Families in green: family known to host at least one vocal species but not yet recorded in French Polynesia. Families in blue: no information concerning acoustic communication. Numbers after each family refer to the number of genera found in Moorea. The Table has been built on the basis of different studies (FISH et al. 1952; Moulton 1958, 1963; Fish \& MOWBRAY 1970; Lobel et al. 2010; MALAVASI et al. 2012; TRICAS \& Boyle 2014; Fine \& PARMENTIER 2015; PARMENTIER et al. 2016).

\begin{tabular}{|c|c|c|c|c|}
\hline FAMILY & GENUS-SPECIES & VSM & VG & PVG \\
\hline \multirow[t]{3}{*}{ Megalopidae } & 1 & & & \\
\hline & Megalops & & & \\
\hline & Megalops cyprinoides & & & \\
\hline \multirow[t]{3}{*}{ Albulidae } & 1 & & & \\
\hline & Albula & & & \\
\hline & Albula glossodonta & & & \\
\hline \multirow[t]{3}{*}{ Chanidae } & 1 & & & \\
\hline & Chanos & & & \\
\hline & Chanos chanos & & & \\
\hline \multirow{3}{*}{ Moringuidae } & 1 & & & \\
\hline & Moringua & & & \\
\hline & Moringua javanica & & & \\
\hline \multirow[t]{6}{*}{ Chlopsidae } & 2 & & & \\
\hline & Chilorhinus & & & \\
\hline & Chilorhinus platyrhynchus & & & \\
\hline & Kaupichthys & & & \\
\hline & Kaupichthys brachychirus & & & \\
\hline & Kaupichthys diodontus & & & \\
\hline \multirow[t]{13}{*}{ Muraenidae } & 8 & & & \\
\hline & Anarchias & & & \\
\hline & Anarchias seychellensis & & & \\
\hline & Echidna & & & \\
\hline & Echidna leucotaenia & & & \\
\hline & Echidna nebulosa & & & \\
\hline & Echidna polyzona & & & \\
\hline & Enchelycore & & & \\
\hline & Enchelycore bayeri & & & \\
\hline & Enchelynassa & & & \\
\hline & Enchelynassa canina & & & \\
\hline & Gymnomuraena & & & \\
\hline & Gymnomuraena zebra & & & \\
\hline
\end{tabular}




\section{Gymnothorax}

Gymnothorax buroensis

Gymnothorax chilospilus

Gymnothorax eurostus

Gymnothorax fimbriatus

Gymnothorax formosus

Gymnothorax gracilicauda

Gymnothorax javanicus

Gymnothorax kontodontos

Gymnothorax margaritophorus

Gymnothorax melatremus

Gymnothorax meleagris

Gymnothorax pictus

Gymnothorax reevesii

Gymnothorax richardsonii

Gymnothorax rueppelliae

Gymnothorax undulatus

Gymnothorax zonipectis

\section{Scuticaria}

Scuticaria tigrina

\section{Uropterygius}

Uropterygius alboguttatus

Uropterygius fuscoguttatus

Uropterygius inornatus

Uropterygius kamar

Uropterygius supraforatus

\begin{tabular}{ll} 
Congridae & $\mathbf{4}$ \\
& Ariosoma \\
& Ariosoma fasciatum \\
& Ariosoma scheelei \\
& Conger \\
& Conger cinereus \\
& Gorgasia \\
& Gorgasia galzini \\
& Heteroconger \\
& Heteroconger lentiginosus \\
& $\mathbf{6}$ \\
\hline Oallechelys \\
Callechelys marmorata
\end{tabular}




\begin{tabular}{|c|c|c|c|c|}
\hline FAMILY & GENUS-SPECIES & VSM & VG & PVG \\
\hline & $\begin{array}{l}\text { Leiuranus } \\
\text { Leiuranus semicinctus } \\
\text { Myrophis } \\
\text { Myrophis microchir } \\
\text { Schismorhynchus } \\
\text { Schismorhynchus labialis } \\
\text { Schultzidia } \\
\text { Schultzidia johnstonensis } \\
\text { Scolecenchelys } \\
\text { Scolecenchelys gymnota } \\
\text { Scolecenchelys laticaudata }\end{array}$ & & & \\
\hline Synodontidae & $\begin{array}{l}2 \\
\text { Saurida } \\
\text { Saurida gracilis } \\
\text { Synodus } \\
\text { Synodus binotatus } \\
\text { Synodus dermatogenys } \\
\text { Synodus jaculum } \\
\text { Synodus variegatus }\end{array}$ & & & \\
\hline Antennariidae & $\begin{array}{l}2 \\
\text { Antennarius } \\
\text { Antennarius randalli } \\
\text { Antennatus } \\
\text { Antennatus coccineus } \\
\text { Antennatus tuberosus }\end{array}$ & & & \\
\hline Ophidiidae & $\begin{array}{l}1 \\
\text { Brotula } \\
\text { Brotula multibarbata }\end{array}$ & & & $\mathrm{X}$ \\
\hline Bythitidae & $\begin{array}{l}3 \\
\text { Alionematichthys } \\
\text { Alionematichthys piger } \\
\text { Diancistrus } \\
\text { Diancistrus katrineae } \\
\text { Dinematichthys } \\
\text { Dinematichthys iluocoeteoides }\end{array}$ & & & $\mathrm{X}$ \\
\hline Carapidae & $\begin{array}{l}3 \\
\text { Carapus } \\
\text { Carapus boraborensis } \\
\text { Carapus homei }\end{array}$ & $\begin{array}{l}X \\
X\end{array}$ & $\mathrm{X}$ & \\
\hline
\end{tabular}




\begin{tabular}{|c|c|c|c|c|}
\hline \multirow[t]{7}{*}{ FAMILY } & GENUS-SPECIES & VSM & VG & PVG \\
\hline & Carapus mourlani & $\mathrm{X}$ & & \\
\hline & Encheliophis & & $\mathrm{X}$ & \\
\hline & Encheliophis chardewalli & $\mathrm{X}$ & & \\
\hline & Encheliophis gracilis & $\mathrm{X}$ & & \\
\hline & Onuxodon & & $X$ & \\
\hline & Onuxodon fowleri & $\mathrm{X}$ & & \\
\hline \multirow[t]{5}{*}{ Hemiramphidae } & 2 & & & \\
\hline & Hemiramphus & & & \\
\hline & Hemiramphus depauperatus & & & \\
\hline & Hyporhamphus & & $\mathrm{X}$ & \\
\hline & Hyporhamphus affinis & & & \\
\hline \multirow[t]{7}{*}{ Belonidae } & 3 & & & \\
\hline & Ablennes & & & \\
\hline & Ablennes hians & & & \\
\hline & Platybelone & & & \\
\hline & Platybelone argalus & & & \\
\hline & Tylosurus & & & \\
\hline & Tylosurus crocodilus & & & \\
\hline \multirow[t]{21}{*}{ Holocentridae } & 4 & & & \\
\hline & Myripristis & & $\mathrm{X}$ & \\
\hline & Myripristis adusta & & & \\
\hline & Myripristis amaena & $\mathrm{X}$ & & \\
\hline & Myripristis berndti & $\mathrm{X}$ & & \\
\hline & Myripristis kuntee & $\mathrm{X}$ & & \\
\hline & Myripristis murdjan & & & \\
\hline & Myripristis pralinia & $\mathrm{X}$ & & \\
\hline & Myripristis violacea & $\mathrm{X}$ & & \\
\hline & Myripristis woodsi & & & \\
\hline & Neoniphon & & $X$ & \\
\hline & Neoniphon argenteus & & & \\
\hline & Neoniphon opercularis & & & \\
\hline & Neoniphon sammara & $\mathrm{X}$ & & \\
\hline & Plectrypops & & & $X$ \\
\hline & Plectrypops lima & & & \\
\hline & Sargocentron & & $\mathrm{X}$ & \\
\hline & Sargocentron caudimaculatum & & & \\
\hline & Sargocentron diadema & & & \\
\hline & Sargocentron microstoma & & & \\
\hline & Sargocentron punctatissimum & & & \\
\hline
\end{tabular}




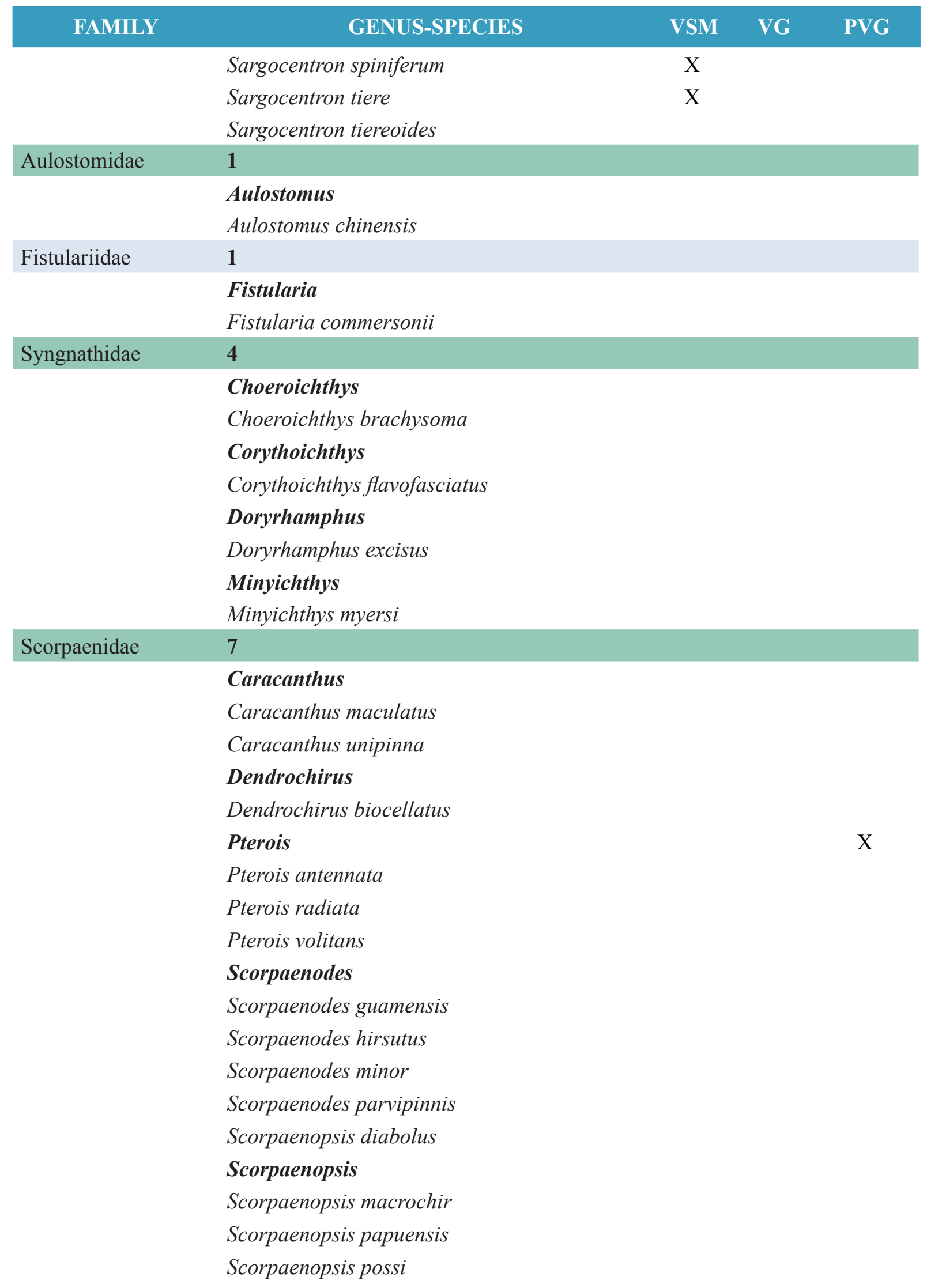




\section{FAMILY}

\section{Sebastapistes}

Sebastapistes fowleri

Sebastapistes strongia

Sebastapistes tinkhami

\section{Taenianotus}

Taenianotus triacanthus

$\begin{array}{ll}\text { Synanceiidae } & \mathbf{1} \\ & \text { Synanceia } \\ & \text { Synanceia verrucosa } \\ \text { Platycephalidae } & \mathbf{1} \\ & \begin{array}{l}\text { Sunagocia } \\ \text { Sunagocia otaitensis }\end{array} \\ \text { Dactylopteridae } & \mathbf{1}\end{array}$

\section{Dactyloptena}

Dactyloptena orientalis

Serranidae

\section{1}

Aporops

Aporops bilinearis

Cephalopholis

Cephalopholis argus

Cephalopholis aurantia

Cephalopholis leopardus

Cephalopholis urodeta

Epinephelus

Epinephelus fasciatus

Epinephelus merra

Epinephelus polyphekadion

Epinephelus socialis

Epinephelus tauvina

\section{Gracila}

Gracila albomarginata

\section{Grammistes}

Grammistes sexlineatus

Grammistops ocellatus

\section{Liopropoma}

Liopropoma mitratum

Liopropoma pallidum

Liopropoma tonstrinum 


\begin{tabular}{|c|c|c|c|c|}
\hline FAMILY & GENUS-SPECIES & VSM & VG & PVG \\
\hline & $\begin{array}{l}\text { Plectranthias } \\
\text { Plectranthias cf. winniensis } \\
\text { Plectranthias longimanus } \\
\text { Plectranthias nanus } \\
\text { Pseudanthias } \\
\text { Pseudanthias bicolor } \\
\text { Pseudanthias lori } \\
\text { Pseudanthias mooreanus } \\
\text { Pseudanthias olivaceus } \\
\text { Pseudanthias pascalus } \\
\text { Pseudogramma } \\
\text { Pseudogramma polyacantha } \\
\text { Suttonia } \\
\text { Suttonia lineata } \\
\text { Variola } \\
\text { Variola louti }\end{array}$ & $\mathrm{X}$ & $\mathrm{X}$ & \\
\hline Pseudochromidae & $\begin{array}{l}1 \\
\text { Pseudoplesiops } \\
\text { Pseudoplesiops revellei }\end{array}$ & & & \\
\hline Priacanthidae & $\begin{array}{l}2 \\
\text { Heteropriacanthus } \\
\text { Heteropriacanthus cruentatus } \\
\text { Priacanthus } \\
\text { Priacanthus hamrur }\end{array}$ & $\mathrm{X}$ & $\begin{array}{l}X \\
X\end{array}$ & \\
\hline Cirrhitidae & $\begin{array}{l}\mathbf{5} \\
\text { Amblycirrhitus } \\
\text { Amblycirrhitus bimacula } \\
\text { Cirrhitichthys } \\
\text { Cirrhitichthys oxycephalus } \\
\text { Cirrhitus } \\
\text { Cirrhitus pinnulatus } \\
\text { Neocirrhites } \\
\text { Neocirrhites armatus } \\
\text { Paracirrhites } \\
\text { Paracirrhites arcatus } \\
\text { Paracirrhites forsteri } \\
\text { Paracirrhites hemistictus }\end{array}$ & & & \\
\hline
\end{tabular}




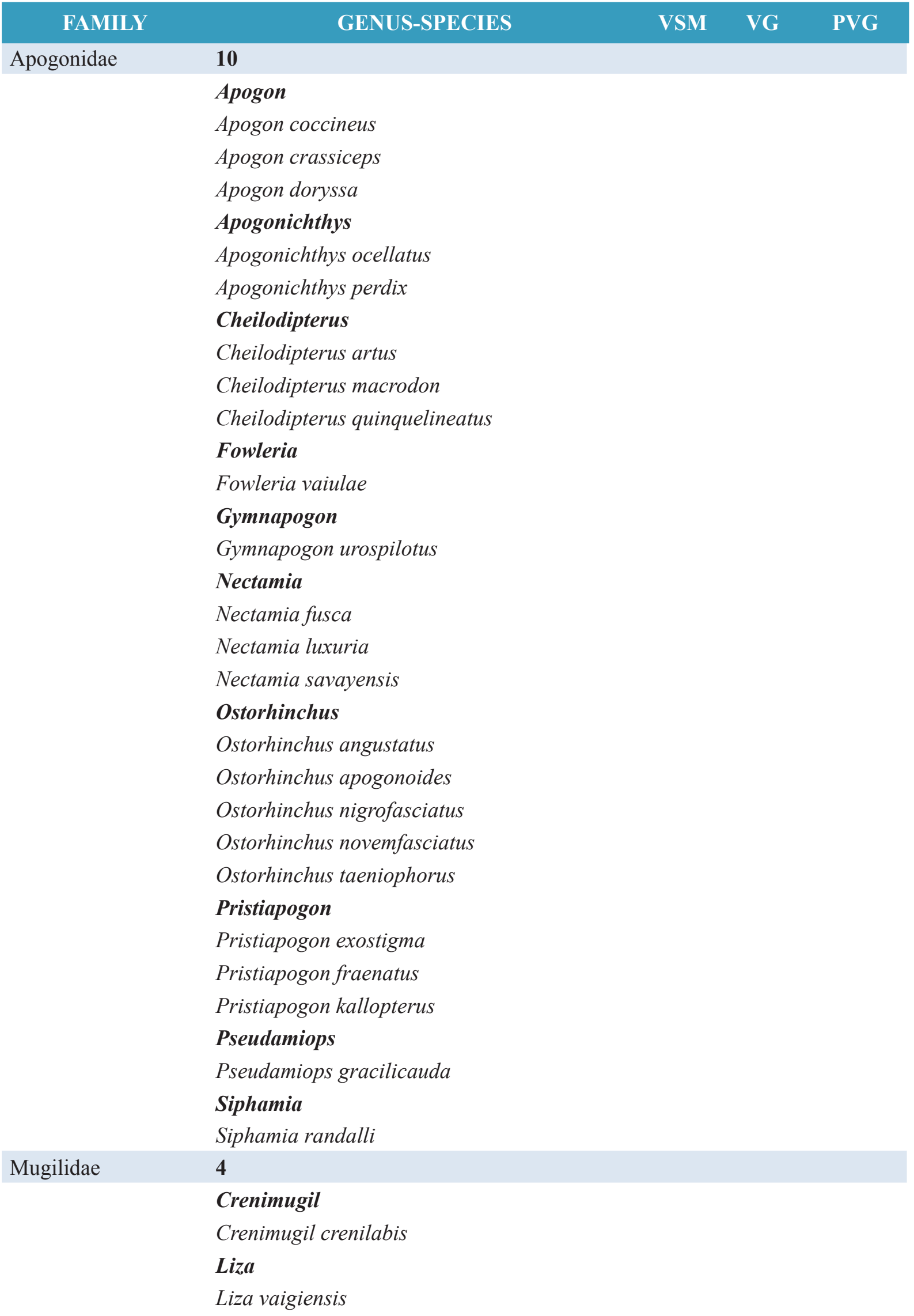




\begin{tabular}{|c|c|c|c|c|}
\hline FAMILY & GENUS-SPECIES & VSM & VG & PVG \\
\hline & $\begin{array}{l}\text { Mugil } \\
\text { Mugil cephalus } \\
\text { Osteomugil } \\
\text { Osteomugil engeli }\end{array}$ & & & \\
\hline \multirow[t]{2}{*}{ Sphyraenidae } & 1 & & & \\
\hline & $\begin{array}{l}\text { Sphyraena } \\
\text { Sphyraena barracuda } \\
\text { Sphyraena forsteri } \\
\text { Sphyraena helleri } \\
\text { Sphyraena qenie }\end{array}$ & & & \\
\hline \multirow[t]{2}{*}{ Polynemidae } & 1 & $\mathbf{X}$ & & \\
\hline & $\begin{array}{l}\text { Polydactylus } \\
\text { Polydactylus plebeius } \\
\text { Polydactylus sexfilis }\end{array}$ & & $\mathrm{X}$ & \\
\hline \multirow[t]{2}{*}{ Malacanthidae } & 1 & & & \\
\hline & $\begin{array}{l}\text { Malacanthus } \\
\text { Malacanthus brevirostris }\end{array}$ & & & \\
\hline \multirow[t]{2}{*}{ Echeneididae } & 1 & & & \\
\hline & $\begin{array}{l}\text { Echeneis } \\
\text { Echeneis naucrates }\end{array}$ & & & \\
\hline \multirow[t]{2}{*}{ Carangidae } & 8 & $\mathbf{X}$ & & \\
\hline & $\begin{array}{l}\text { Carangoides } \\
\text { Carangoides ferdau } \\
\text { Carangoides orthogrammus } \\
\text { Caranx } \\
\text { Caranx ignobilis } \\
\text { Caranx lugubris } \\
\text { Caranx melampygus } \\
\text { Caranx papuensis } \\
\text { Caranx sexfasciatus } \\
\text { Decapterus } \\
\text { Decapterus macarellus } \\
\text { Decapterus macrosoma } \\
\text { Elagatis } \\
\text { Elagatis bipinnulata } \\
\text { Scomberoides } \\
\text { Scomberoides lysan } \\
\text { Selar } \\
\text { Selar crumenophthalmus }\end{array}$ & & $X$ & \\
\hline
\end{tabular}




\section{FAMILY}

GENUS-SPECIES

VSM

VG

PVG

Seriola

Seriola rivoliana

Trachinotus

$\mathrm{X}$

Trachinotus baillonii

$\begin{array}{ll}\text { Lutjanidae } & \mathbf{4} \\ & \text { Aphareus } \\ & \text { Aphareus furca } \\ & \text { Aprion } \\ \text { Aprion virescens }\end{array}$

\section{Lutjanus}

Lutjanus bohar

Lutjanus fulvus

Lutjanus gibbus

Lutjanus kasmira

X

Lutjanus monostigma

Lutjanus rivulatus

Paracaesio

Paracaesio xanthura

\begin{tabular}{|c|c|c|c|}
\hline \multirow[t]{3}{*}{ Caesionidae } & 1 & $\mathbf{X}$ & \\
\hline & Pterocaesio & & $\mathrm{X}$ \\
\hline & Pterocaesio tile & & \\
\hline \multirow[t]{8}{*}{ Lethrinidae } & 3 & & \\
\hline & Gnathodentex & & \\
\hline & Gnathodentex aureolineatus & & \\
\hline & Lethrinus & & \\
\hline & Lethrinus olivaceus & & \\
\hline & Lethrinus xanthochilus & & \\
\hline & Monotaxis & & \\
\hline & Monotaxis grandoculis & & \\
\hline \multirow[t]{11}{*}{ Mullidae } & 3 & & \\
\hline & Mulloidichthys & & $\mathrm{X}$ \\
\hline & Mulloidichthys flavolineatus & $\mathrm{X}$ & \\
\hline & Mulloidichthys vanicolensis & & \\
\hline & Parupeneus & & $\mathrm{X}$ \\
\hline & Parupeneus barberinus & & \\
\hline & Parupeneus ciliatus & & \\
\hline & Parupeneus cyclostomus & & \\
\hline & Parupeneus insularis & $\mathrm{X}$ & \\
\hline & Parupeneus multifasciatus & $\mathrm{X}$ & \\
\hline & Parupeneus pleurostigma & & \\
\hline
\end{tabular}




\begin{tabular}{|c|c|c|c|c|}
\hline FAMILY & GENUS-SPECIES & VSM & VG & PVG \\
\hline & Upeneus & & & \\
\hline & Upeneus vittatus & & & \\
\hline \multirow[t]{3}{*}{ Pempherididae } & 1 & & & \\
\hline & Pempheris & & $\mathrm{X}$ & \\
\hline & Pempheris oualensis & $\mathrm{X}$ & & \\
\hline \multirow[t]{4}{*}{ Kyphosidae } & 1 & $\mathbf{X}$ & & \\
\hline & Kyphosus & & $X$ & \\
\hline & Kyphosus cinerascens & & & \\
\hline & Kyphosus vaigiensis & & & \\
\hline \multirow[t]{30}{*}{ Chaetodontidae } & 4 & & & \\
\hline & Chaetodon & & $\mathrm{X}$ & \\
\hline & Chaetodon auriga & $\mathrm{X}$ & & \\
\hline & Chaetodon bennetti & & & \\
\hline & Chaetodon citrinellus & & & \\
\hline & Chaetodon ephippium & & & \\
\hline & Chaetodon lineolatus & & & \\
\hline & Chaetodon lunula & & & \\
\hline & Chaetodon lunulatus & & & \\
\hline & Chaetodon mertensii & & & \\
\hline & Chaetodon ornatissimus & $\mathrm{X}$ & & \\
\hline & Chaetodon pelewensis & & & \\
\hline & Chaetodon quadrimaculatus & & & \\
\hline & Chaetodon reticulatus & & & \\
\hline & Chaetodon semeion & & & \\
\hline & Chaetodon trichrous & & & \\
\hline & Chaetodon trifascialis & & & \\
\hline & Chaetodon ulietensis & & & \\
\hline & Chaetodon unimaculatus & & & \\
\hline & Chaetodon vagabundus & & & \\
\hline & Forcipiger & & $\mathrm{X}$ & \\
\hline & Forcipiger flavissimus & $\mathrm{X}$ & & \\
\hline & Forcipiger longirostris & $\mathrm{X}$ & & \\
\hline & Hemitaurichthys & & $\mathrm{X}$ & \\
\hline & Hemitaurichthys polylepis & $\mathrm{X}$ & & \\
\hline & Hemitaurichthys thompsoni & $\mathrm{X}$ & & \\
\hline & Heniochus & & $X$ & \\
\hline & Heniochus acuminatus & & & \\
\hline & Heniochus chrysostomus & $\mathrm{x}$ & & \\
\hline & Heniochus monoceros & & & \\
\hline
\end{tabular}




\begin{tabular}{|c|c|c|c|c|}
\hline FAMILY & GENUS-SPECIES & VSM & VG & PVG \\
\hline \multirow[t]{3}{*}{ Ephippididae } & 1 & $\mathbf{X}$ & & \\
\hline & Platax & & $\mathrm{X}$ & \\
\hline & Platax orbicularis & & & \\
\hline \multirow[t]{17}{*}{ Pomacanthidae } & 5 & & & \\
\hline & Centropyge & & $\mathrm{X}$ & \\
\hline & Centropyge bispinosa & & & \\
\hline & Centropyge fisheri & & & \\
\hline & Centropyge flavissima & & & \\
\hline & Centropyge heraldi & & & \\
\hline & Centropyge loricula & $\mathrm{X}$ & & \\
\hline & Centropyge multicolor & & & \\
\hline & Genicanthus & & & \\
\hline & Genicanthus bellus & & & \\
\hline & Genicanthus watanabei & & & \\
\hline & Paracentropyge & & & \\
\hline & Paracentropyge multifasciata & & & \\
\hline & Pomacanthus & & $\mathrm{X}$ & \\
\hline & Pomacanthus imperator & $\mathrm{X}$ & & \\
\hline & Pygoplites & & & \\
\hline & Pygoplites diacanthus & & & \\
\hline \multirow[t]{18}{*}{ Pomacentridae } & 10 & & & \\
\hline & Abudefduf & $\mathrm{X}$ & $X$ & \\
\hline & Abudefduf septemfasciatus & & & \\
\hline & Abudefduf sexfasciatus & & & \\
\hline & Abudefduf sordidus & & & \\
\hline & Abudefduf vaigiensis & $\mathrm{X}$ & & \\
\hline & Amphiprion & & $\mathrm{X}$ & \\
\hline & Amphiprion cf. chrysopterus & & & \\
\hline & Chromis & & $\mathrm{X}$ & \\
\hline & Chromis acares & & & \\
\hline & Chromis agilis & $\mathrm{X}$ & & \\
\hline & Chromis alpha & & & \\
\hline & Chromis atripectoralis & & & \\
\hline & Chromis iomelas & & & \\
\hline & Chromis margaritifer & & & \\
\hline & Chromis vanderbilti & $\mathrm{X}$ & & \\
\hline & Chromis viridis & & & \\
\hline & Chromis xanthura & & & \\
\hline
\end{tabular}




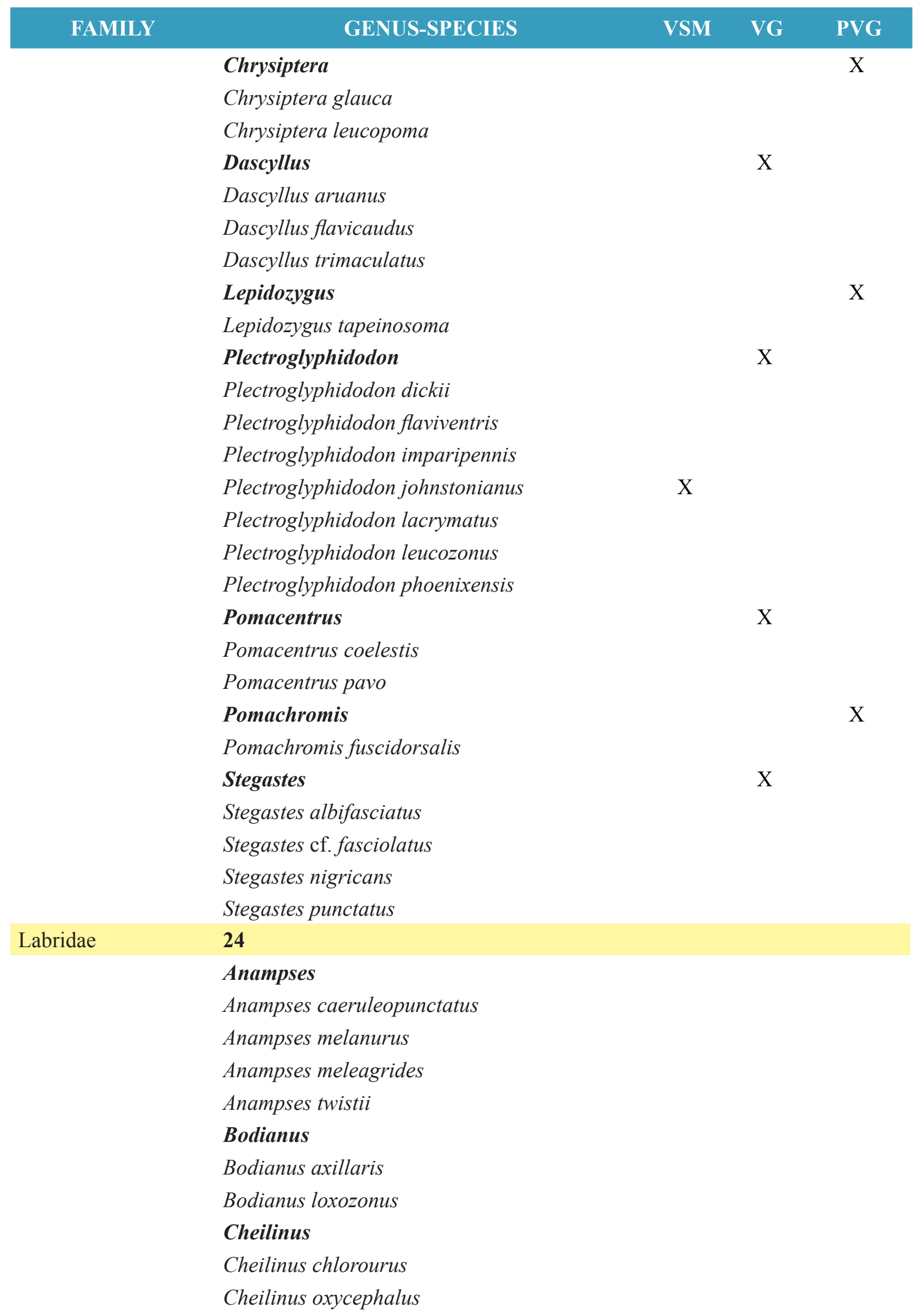


Cheilinus trilobatus

Cheilinus undulatus

Cheilio inermis

\section{Cirrhilabrus}

Cirrhilabrus exquisitus

Cirrhilabrus scottorum

\section{Coris}

Coris aygula

Coris gaimard

Cymolutes

Cymolutes praetextatus

\section{Epibulus}

Epibulus insidiator

\section{Gomphosus}

Gomphosus varius

\section{Halichoeres}

Halichoeres claudia

Halichoeres hortulanus

Halichoeres margaritaceus

Halichoeres marginatus

Halichoeres melasmapomus

Halichoeres trimaculatus

\section{Hemigymnus}

Hemigymnus fasciatus

Hologymnosus

Hologymnosus annulatus

Hologymnosus doliatus

\section{Iniistius}

Iniistius pavo

Labroides

Labroides bicolor

Labroides dimidiatus

Labroides rubrolabiatus

\section{Labropsis}

Labropsis polynesica

Macropharyngodon

Macropharyngodon meleagris

Novaculichthys

Novaculichthys taeniourus 


\section{FAMILY}

\section{Oxycheilinus}

Oxycheilinus bimaculatus

Oxycheilinus digramma

Oxycheilinus unifasciatus

Pseudocheilinus

Pseudocheilinus evanidus

Pseudocheilinus hexataenia

Pseudocheilinus ocellatus

Pseudocheilinus octotaenia

Pseudocheilinus tetrataenia

Pseudocoris

Pseudocoris aurantiofasciata

\section{Pseudodax}

Pseudodax moluccanus

Pseudojuloides

Pseudojuloides atavai

Pseudojuloides cerasinus

\section{Stethojulis}

Stethojulis bandanensis

Stethojulis strigiventer

Thalassoma

Thalassoma amblycephalum

Thalassoma hardwicke

Thalassoma lutescens

Thalassoma purpureum

Thalassoma quinquevittatum

Thalassoma trilobatum

Wetmorella

Wetmorella albofasciata

Wetmorella nigropinnata

Scaridae 6

\section{Calotomus}

Calotomus carolinus

\section{Cetoscarus}

Cetoscarus ocellatus

Chlorurus

Chlorurus frontalis

Chlorurus microrhinos

Chlorurus spilurus 


\section{FAMILY}

Hipposcarus

Hipposcarus longiceps

Leptoscarus

Leptoscarus vaigiensis

Scarus

Scarus altipinnis

Scarus chameleon

Scarus festivus

Scarus forsteni

Scarus frenatus

Scarus ghobban

Scarus globiceps

Scarus longipinnis

Scarus niger

Scarus oviceps

Scarus psittacus

Scarus rubroviolaceus

Scarus schlegeli

$\begin{array}{ll}\text { Pinguipedidae } & \mathbf{1} \\ & \text { Parapercis } \\ & \text { Parapercis millepunctata } \\ & \text { Parapercis schauinslandii } \\ \text { Creediidae } & \mathbf{2} \\ & \text { Chalixodytes } \\ & \text { Chalixodytes tauensis } \\ & \text { Limnichthys } \\ & \text { Limnichthys nitidus } \\ & \mathbf{2} \\ \text { Trypterygiidae } & \text { Enneapterygius } \\ & \text { Enneapterygius fuscoventer } \\ & \text { Enneapterygius hemimelas } \\ & \text { Enneapterygius pyramis } \\ & \text { Helcogramma } \\ & \text { Helcogramma capidata } \\ & \mathbf{1 3} \\ \text { Aspidontus } \\ \text { Aspidontus dussumieri } \\ \text { Aspidontus taeniatus } \\ \text { Atrosalarias } \\ \text { Atrosalarias fuscus } \\ \end{array}$




\section{FAMILY}

\section{Blenniella}

Blenniella gibbifrons

\section{Cirripectes}

Cirripectes fuscoguttatus

Cirripectes quagga

Cirripectes variolosus

Enchelyurus

Enchelyurus ater

\section{Entomacrodus}

Entomacrodus cymatobiotus

Entomacrodus thalassinus

\section{Exallias}

Exallias brevis

\section{Glyptoparus}

Glyptoparus delicatulus

\section{Istiblennius}

Istiblennius edentulus

\section{Nannosalarias}

Nannosalarias nativitatis

\section{Petroscirtes}

Petroscirtes xestus

\section{Plagiotremus}

Plagiotremus tapeinosoma

\section{Stanulus}

Stanulus seychellensis

$\begin{array}{ll}\text { Callionymidae } & \mathbf{2} \\ & \text { Callionymus } \\ & \text { Callionymus filamentosus } \\ & \text { Callionymus simplicicornis } \\ & \text { Diplogrammus } \\ & \text { Diplogrammus goramensis } \\ & \mathbf{2 2} \\ \text { Gobiidae } & \text { Amblyeleotris } \\ & \text { Amblyeleotris fasciata } \\ & \text { Amblygobius } \\ & \text { Amblygobius nocturnus } \\ & \text { Asterropteryx } \\ & \text { Asterropteryx ensifera } \\ & \text { Asterropteryx semipunctata }\end{array}$




\section{Bathygobius}

Bathygobius coalitus

Bathygobius cotticeps

Bryaninops

Bryaninops tigris

Bryaninops yongei

\section{Cabillus}

Cabillus tongarevae

\section{Callogobius}

Callogobius sclateri

\section{Ctenogobiops}

Ctenogobiops feroculus

Eviota

Eviota afelei

Eviota albolineata

Eviota disrupta

Eviota infulata

Eviota prasites

\section{Fusigobius}

Fusigobius duospilus

Fusigobius humeralis

Fusigobius neophytus

\section{Gnatholepis}

Gnatholepis anjerensis

Gnatholepis cauerensis

\section{Gobiodon}

Gobiodon quinquestrigatus

\section{Gobiopsis}

Gobiopsis exigua

Oligolepis

Oligolepis cf. pacificus (Moorea Is.)

\section{Oplopomus}

Oplopomus caninoides

Oplopomus oplopomus

\section{Paragobiodon}

Paragobiodon echinocephalus

Paragobiodon lacunicolus

Paragobiodon modestus 


\section{FAMILY}

Pleurosicya

Pleurosicya labiata

Pleurosicya micheli

Pleurosicya mossambica

Priolepis

Priolepis ailina

Priolepis cincta

Priolepis compita

Priolepis inhaca

Priolepis semidoliata

Priolepis squamogena

Priolepis triops

\section{Trimma}

Trimma emeryi

Trimma milta

\section{Trimmatom}

Trimmatom nanus

\section{Valenciennea}

Valenciennea strigata

\section{Vanderhorstia}

Vanderhorstia ornatissima

Vanderhorstia steelei

$\begin{array}{ll}\text { Eleotridae } & \mathbf{1} \\ & \text { Calumia } \\ \text { Calumia godeffroyi } & \mathbf{3} \\ \text { Munnellichthys } \\ \text { Gunnellichthys curiosus } \\ \text { Gunnellichthys monostignadae } \\ \text { Nemateleotris } \\ \text { Nemateleotris helfrichi } \\ \text { Nemateleotris magnifica } \\ \text { Ptereleotris } \\ \text { Ptereleotris evides } \\ \text { Ptereleotris hanae } \\ \text { Ptereleotris heteroptera } \\ \text { Ptereleotris microlepis } \\ \text { Ptereleotris monoptera } \\ \text { Ptereleotris zebra }\end{array}$




\begin{tabular}{|c|c|c|c|c|}
\hline FAMILY & GENUS-SPECIES & VSM & VG & PVG \\
\hline \multirow[t]{3}{*}{ Scombridae } & 1 & & & \\
\hline & Gymnosarda & & & \\
\hline & Gymnosarda unicolor & & & \\
\hline \multirow[t]{3}{*}{ Zanclidae } & 1 & & & \\
\hline & Zanclus & & $X$ & \\
\hline & Zanclus cornutus & $\mathrm{X}$ & & \\
\hline \multirow[t]{33}{*}{ Acanthuridae } & 4 & & & \\
\hline & Acanthurus & & $\mathrm{X}$ & \\
\hline & Acanthurus achilles & & & \\
\hline & Acanthurus blochii & & & \\
\hline & Acanthurus guttatus & & & \\
\hline & Acanthurus leucopareius & & & \\
\hline & Acanthurus lineatus & & & \\
\hline & Acanthurus mata & & & \\
\hline & Acanthurus nigricans & & & \\
\hline & Acanthurus nigricauda & & & \\
\hline & Acanthurus nigrofuscus & & & \\
\hline & Acanthurus nigros & & & \\
\hline & Acanthurus nubilus & & & \\
\hline & Acanthurus olivaceus & $\mathrm{X}$ & & \\
\hline & Acanthurus pyroferus & & & \\
\hline & Acanthurus thompsoni & & & \\
\hline & Acanthurus triostegus & & & \\
\hline & Acanthurus xanthopterus & & & \\
\hline & Ctenochaetus & & $\mathrm{X}$ & \\
\hline & Ctenochaetus binotatus & & & \\
\hline & Ctenochaetus flavicauda & & & \\
\hline & Ctenochaetus hawaiiensis & $\mathrm{X}$ & & \\
\hline & Ctenochaetus striatus & & & \\
\hline & Naso & & & \\
\hline & Naso annulatus & & & \\
\hline & Naso brachycentron & & & \\
\hline & Naso brevirostris & & & \\
\hline & Naso hexacanthus & & & \\
\hline & Naso lituratus & & & \\
\hline & Naso unicornis & & & \\
\hline & Naso vlamingii & & & \\
\hline & Zebrasoma & & $\mathrm{X}$ & \\
\hline & Zebrasoma rostratum & & & \\
\hline
\end{tabular}




\begin{tabular}{|c|c|c|c|c|}
\hline FAMILY & GENUS-SPECIES & VSM & VG & PVG \\
\hline & Zebrasoma scopas & & & \\
\hline & Zebrasoma velifer & & & \\
\hline \multirow[t]{4}{*}{ Siganidae } & 1 & & & \\
\hline & Siganus & & & \\
\hline & Siganus argenteus & & & \\
\hline & Siganus spinus & & & \\
\hline \multirow[t]{6}{*}{ Bothidae } & 2 & & & \\
\hline & Asterorhombus & & & \\
\hline & Asterorhombus filifer & & & \\
\hline & Bothus & & & \\
\hline & Bothus mancus & & & \\
\hline & Bothus pantherinus & & & \\
\hline \multirow[t]{3}{*}{ Samaridae } & 1 & & & \\
\hline & Samariscus & & & \\
\hline & Samariscus triocellatus & & & \\
\hline \multirow[t]{3}{*}{ Soleidae } & 1 & & & \\
\hline & Aseraggodes & & & \\
\hline & Aseraggodes melanostictus & & & \\
\hline \multirow[t]{22}{*}{ Balistidae } & 8 & & & \\
\hline & Balistapus & & $\mathrm{X}$ & \\
\hline & Balistapus undulatus & $\mathrm{X}$ & & \\
\hline & Balistoides & & & \\
\hline & Balistoides viridescens & & & \\
\hline & Melichthys & & $\mathrm{X}$ & \\
\hline & Melichthys niger & $\mathrm{X}$ & & \\
\hline & Melichthys vidua & $\mathrm{X}$ & & \\
\hline & Odonus & & $X$ & \\
\hline & Odonus niger & $\mathrm{X}$ & & \\
\hline & Pseudobalistes & & & \\
\hline & Pseudobalistes flavimarginatus & & & \\
\hline & Pseudobalistes fuscus & & & \\
\hline & Rhinecanthus & & $X$ & \\
\hline & Rhinecanthus aculeatus & $\mathrm{X}$ & & \\
\hline & Rhinecanthus lunula & & & \\
\hline & Rhinecanthus rectangulus & $\mathrm{X}$ & & \\
\hline & Sufflamen & & $\mathrm{X}$ & \\
\hline & Sufflamen bursa & $\mathrm{X}$ & & \\
\hline & Sufflamen fraenatum & $\mathrm{X}$ & & \\
\hline & Xanthichthys & & $\mathrm{X}$ & \\
\hline & Xanthichthys auromarginatus & $\mathrm{X}$ & & \\
\hline
\end{tabular}




\begin{tabular}{|c|c|c|c|c|}
\hline FAMILY & GENUS-SPECIES & VSM & VG & PVG \\
\hline \multirow[t]{10}{*}{ Monacanthidae } & 4 & & & \\
\hline & Aluterus & & $\mathrm{X}$ & \\
\hline & Aluterus scriptus & $\mathrm{X}$ & & \\
\hline & Amanses & & & \\
\hline & Amanses scopas & & & \\
\hline & Cantherhines & & $\mathrm{X}$ & \\
\hline & Cantherhines dumerilii & & & \\
\hline & Cantherhines sandwichiensis & & & \\
\hline & Pervagor & & & \\
\hline & Pervagor aspricaudus & & & \\
\hline \multirow[t]{6}{*}{ Ostraciidae } & 2 & & & \\
\hline & Lactoria & & $\mathrm{X}$ & \\
\hline & Lactoria cornuta & $\mathrm{X}$ & & \\
\hline & Ostracion & & $\mathrm{X}$ & \\
\hline & Ostracion cubicus & $\mathrm{x}$ & & \\
\hline & Ostracion meleagris & $\mathrm{X}$ & & \\
\hline \multirow[t]{11}{*}{ Tetraodontidae } & 2 & & & \\
\hline & Arothron & & & \\
\hline & Arothron hispidus & & & \\
\hline & Arothron meleagris & & & \\
\hline & Arothron stellatus & & & \\
\hline & Canthigaster & & & \\
\hline & Canthigaster amboinensis & & & \\
\hline & Canthigaster bennetti & & & \\
\hline & Canthigaster janthinoptera & & & \\
\hline & Canthigaster solandri & & & \\
\hline & Canthigaster valentini & & & \\
\hline \multirow[t]{4}{*}{ Diodontidae } & 1 & $\mathrm{X}$ & & \\
\hline & Diodon & & & $\mathrm{X}$ \\
\hline & Diodon hystrix & & & \\
\hline & Diodon liturosus & & & \\
\hline
\end{tabular}




\section{Acknowledgements}

We thank Dr J.-P. Lagardère, Dr R. Rountree and M.L. Fine for their constructive comments. The authors of this paper wish to acknowledge the contributions, funding and support that the work has received: Ministère de l'Économie verte et du domaine - Délégation à la recherche de Polynésie française (contrat N3622 MED-EPHE), Fondation de France (2019-08602), LabEx CORAIL (project EMUL), ANR (project MANINI) and PDR (T.0192.20) from the Belgian National Fund for Scientific Research (FRSFNRS).

\section{Conflict of interest}

The authors have declared no conflicts of interests.

\section{References}

AMORIM M.C.P., VASCONCElos R.O. \& FonseCA P.J. (2015). Fish sounds and mate choice. In: LADICH F. (ed.) Sound Communication in Fishes: 1-33. Springer, Vienna. https://doi.org/10.1007/978-3-7091-1846-7_1

Bertucci F., Maratrat K., Berthe C., Besson M., Guerra A.S., Raick X., Lerouvreur F., LECCHINI D. \& PARMENTIER E. (2020). Local sonic activity reveals potential partitioning in a coral reef fish community. Oecologia 193: 125-134. https://doi.org/10.1007/s00442-020-04647-3

Bolgan M. \& PARMentier E. (2020). The unexploited potential of listening to deep-sea fish. Fish and Fisheries 21: 1238-1256. https://doi.org/10.1111/faf.12493

Bradbury J.W. \& VehrenCAMP S.L. (1998). Principles of Animal Communication. Sinauer Associates, Inc., Sunderland, Massachusetts.

Connaughton M.A., Fine, M.L. \& TAYlor M.H. (1997). The effects of seasonal hypertrophy and atrophy on fiber morphology, metabolic substrate concentration and sound characteristics of the weakfish sonic muscle. The Journal of Experimental Biology 200: 2449-2457.

Cousteau J.Y. \& Dumas F. (1953). The Silent World. Harper and Brothers, New York.

De Jong K., Bouton N. \& SlabbeKoorn H. (2007). Azorean rock-pool blennies produce sizedependent calls in a courtship context. Animal Behaviour 74: 1285-1292.

https://doi.org/10.1016/j.anbehav.2007.02.023

DesiderÀ E., Guidetti P., Panzalis P., Navone A., Valentini-Poirrier C.-A., Boissery P. \& GERVAISE C. (2019). Acoustic fish communities: sound diversity of rocky habitats reflects fish species diversity . Marine Ecology Progress Series 608: 183-197. https://doi.org/10.3354/meps12812

FInE M.L. \& PARMEnTIER E. (2015). Mechanisms of sound production. In: LADich F. (ed.) Sound Communication in Fishes: 77-126. Springer, Vienna.

FISH M.P. \& MowbraY H.M. (1970). Sounds of Western North Atlantic Fishes. The Johns Hopkins Press, Baltimore.

Fish M.P., KeLSEY A.S.J. \& MowbraY W.H. (1952). Studies on the production of underwater sound by North Atlantic coastal fishes. Journal of Marine Research 11: 180-193.

FréDÉrich B., Olivier D., Litsios G., Alfaro M.E. \& PARMentier E. (2014). Trait decoupling promotes evolutionary diversification of the trophic and acoustic system of damselfishes. Proceedings of the Royal Society B: Biological Sciences 281: 20141047. https://doi.org/10.1098/rspb.2014.1047 
Froese R. \& Pauly D. (2019). FishBase. World Wide Web electronic publication. [Online.] Available from www.fishbase.org [accessed February 2019].

HALLACHER L.E. (1974). The comparative morphology of extrinsic gasbladder musculature in the scorpionfish genus Sebastes (Pisces: Scorpaenidae). Proceedings of the California Academy of Sciences 40: 59-86.

Jublier N., Bertucci F., Kéver L., Colleye O., Ballesta L., Nemeth R.S., LeCChini D., Rhodes K.L. \& PARMENTIER E. (2019). Passive monitoring of phenological acoustic patterns reveals the sound of the camouflage grouper, Epinephelus polyphekadion. Aquatic Conservation: Marine and Freshwater Ecosystems 30: 42-52. https://doi.org/10.1002/aqc.3242

LAGARDĖRE J.P. \& MARIANI A. (2006). Spawning sounds in meagre Argyrosomus regius recorded in the Gironde estuary, France. Journal of Fish Biology 69: 1697-1708.

https://doi.org/10.1111/j.1095-8649.2006.01237.x

LOBEL P.S., KAATZ I.M. \& RicE A.N. (2010). Acoustical behavior of coral reef fishes. In: COLE K.S. (ed.) Reproduction and Sexuality in Marine Fishes: Patterns and Procesess: 307-386. University of California Press, Berkeley, CA. https://doi.org/10.1525/california/9780520264335.003.0010

MalaVasi S., GKenas C., Leonardos I., Torricelli P. \& MCLennAN D.A. (2012). The phylogeny of a reduced "sand goby" group based on behavioural and life history characters. Zoological Journal of the Linnean Society 165: 916-924. https://doi.org/10.1111/j.1096-3642.2012.00832.x

Moulton J.M. (1958). The acoustical behavior of some fishes in the Bimini area. Biological Bulletin 114: 357-374. https://doi.org/10.2307/1538991

Moulton J.M. (1963). Acoustic behaviour of fishes. In: Busnel R.G. (ed.) Acoustic Behaviour of Animals: 687-693. Elsevier Publishing Company, Amsterdam/London/New York.

Parmentier E., Bouillac G., Dragicevic B., Dulcic J. \& Fine M.L. (2010). Call properties and morphology of the sound-producing organ in Ophidion rochei (Ophidiidae). The Journal of Experimental Biology 213: 3230-3236. https://doi.org/10.1242/jeb.044701

Parmentier E., Kéver L., Boyle K., Corbisier Y.-E., SAwelew L. \& Malavasi S. (2013). Sound production mechanism in Gobius paganellus (Gobiidae). The Journal of Experimental Biology 216: 3189-3199. https://doi.org/10.1242/jeb.087205

Parmentier E., LanterbecQ D. \& Eeckhaut I. (2016). From commensalism to parasitism in Carapidae (Ophidiiformes): Heterochronic modes of development? PeerJ 2016: e1786. https://doi.org/10.7717/peerj.1786

Picciulin M., Kéver L., PARmentier E. \& Bolgan M. (2019). Listening to the unseen: passive acoustic monitoring reveals the presence of a cryptic fish species. Aquatic Conservation: Marine and Freshwater Ecosystems 29: 202-210. https://doi.org/10.1002/aqc.2973

Raick X., Lecchini D., Kéver L., Colleye O., Bertucci F. \& Parmentier E. (2018). Sound production mechanism in triggerfish (Balistidae): a synapomorphy. The Journal of Experimental Biology 221: jeb.168948. https://doi.org/10.1242/jeb.168948

Rountree R.A., JuANES F. \& Bolgan M. (2018). Air movement sound production by alewife, white sucker, and four salmonid fishes suggests the phenomenon is widespread among freshwater fishes. PLoS ONE 13: e0204247. https://doi.org/10.1371/journal.pone.0204247

Ruppé L., Clément G., Herrel A., Ballesta L., DéCamps T., Kéver L. \& Parmentier E. (2015). Environmental constraints drive the partitioning of the soundscape in fishes. Proceedings of the National Academy of Sciences 112: 6092-6097. https://doi.org/10.1073/pnas.1424667112 
Siu G., Bacchet P., Bernardi G., Brooks A.J., Carlot J., Causse R., Claudet J., Clua E., Delrieu-Trottin E., Espiau B., Harmelin-Vivien M., Keith P., Lecchini D., Madi Moussa R., Parravicini V., Planes S., Ponsonnet C., Randall J.E., Sasal P., Taquet M., Williams J.T. \& Galzin R. (2017). Shore fishes of French Polynesia. Cybium 41: 245-278. https://doi.org/10.26028/cybium/2017-413-003

TRICAS T.C. \& BOYLE K.S. (2014). Acoustic behaviors in Hawaiian coral reef fish communities. Marine Ecology Progress Series 511: 1-16. https://doi.org/10.3354/meps 10930

VAN DER LAAN R. \& FRICKE R. (2019). Eschmeyer's Catalog of Fishes: Family-Group Names. Available from http://www.calacademy.org/scientists/catalog-of-fishes-family-group-names/

Manuscript received: 6 September 2020

Manuscript accepted: 6 January 2021

Published on: 2 February 2021

Branch editor: Steven Degraer 